Pregented at the Seminer on Symehrotron

Radiation Facilitias, Honolulu, 11/5-9/79

To be published in Nucl. Inst. \&ethods

BNL-27654

ClNi $791176--2$

\title{
Optimization of a Synchrotron Radiation Source*
}

by

A. van Steentergen

National Synchrotron Light Source

Brookhaven National Laboratory, Upton, NY 11973, U.S.A.

\section{Abstract}

Some aspecta of the optimization of an electron storage ring as a synchrotron radiation source will be presented. This will include the choice of magnet ring structure, choice of electron accelersting system parameters and choice of structure sections for the use of high field wigglers or undulators.

*Research supported by the 0.S. Department of Energy. 


\section{Introduction}

The very pronounced growth of the utilization of aynchrotron radiation for research in the domain of the material sciences, atomic and molecular physics, biology and technology has led to the construction of a new generation of high curreat electron stor ge rings dedicated solely to the usage of the enitted aynchrotron radiation. As a consequence it became feasible, in the design stage, to optimize the ring structure, as a multiple photon port gtructure with optimized optical characteristiss, rather than, typically, an optimized structure for $e^{+}-e^{-}$colliding beams dedicated to high energy particle physics research. For example, the deleterious effect due to nonlinear tune shifts asociated with the interaction of the two beams in a colliding beam facility puts a limit on the minimum vartical beam emittance desirable in order to obtain maximum interacting beam luminosity. For a single beam synchrotron radiation source, minimum emittances are desirable in order to obtain optimum source brightness. It is possible to achieve this by optimizing specifically the storage ring lattice structure so that maximum damping (associated with the enission of the synchiotron radiation) of the transverse betatron oscillations occurs.

A1so, in a "conveational" $e^{+}-e^{-}$colliding beam atorage =ing or electron synchrotron, the choice of maximum dipole field value is guided principally by the desire to minimize the total synchrotron radiation power emitted and generally low field values are adopted. With the desire for high flux, high photon energy synchrotron radiation sources it is more fevorable to adopt high dipole field values in the orbit lattice structure. Tnis may be further enhanced by incorporsting very high field (superconducting magnet) devices at a number of specific locations in the storage ring. The inclusion of these so-called orbit wigglers and the optimization of the associated 
syncnrotron radiation sources introduces new constraints on the electron ring lattice structure, which can be wet optimelly only in the basic design of a (dedicated) synchrotron rad1qtion source.

In the following a few specific aspects entering into the optimization of an electron storage ring as a synchrotron radiation source will be dealt with.

Synchrotron Radiation Source Definition

The basic relationship correlating photon source brightness and electron beam parameters has been worked out by Greenl and can be expressed 8s:

$$
\begin{aligned}
& B\left(X=X^{\prime}=Y=Y^{\prime}=0\right)=N_{k}(0, \lambda) / 2 \pi \sigma_{x} \sigma_{y} \\
& N_{k}(0, \lambda)=3.4610^{15} k I \gamma^{2} B_{2}\left(\lambda_{c} / \lambda\right) \\
& \text { with }
\end{aligned}
$$

where $X, X^{\prime}, Y, Y^{\prime}$ refer to the components of the (four dimensionsl) photor: beam phase space and $\sigma_{x}, \sigma_{y}$ are the electron beam transverse dimensions. This expression is valid, in first approximation, for an azimuthally limited length source, at or near a bean "waist" location, with the condition that $\sigma^{\prime}$ $<\sigma_{r}$ and $\sigma_{x^{\prime}}<\sigma_{r}$ where $\sigma_{r} \simeq 0.56 \gamma^{-1}\left(i / \lambda_{c}\right)^{0.43}$, is the natural opening angle of the synchrotron radiation. From this expression it follows that maximum source brightness is obtained for minimum values of $\sigma_{x}, \sigma_{y}\left(\sigma_{x},, \sigma_{y},\right)$ or minimum emittance values $\varepsilon_{x}, \varepsilon_{y}$ of the electron bean (note $\sigma_{x}=\sqrt{\varepsilon_{x}^{\beta}}$ ). 
Although, in general, maximum source brightness is desirable, such as for experiments requiring small angular spread on a small sample, as for example in protein cryatallography, there are a number of experimental arrangementa, whereby only the two dimensional phase space photon flux density is of importance, e.g. whereby with the use of alfts the resolving power of a monochromator is improved. Depending on the use of an imaging element between the synchrotron radiation source and, typically, the monochromator entrance slit, the transmitted photon $f: a$ through the entrance slit is given byl

$$
\begin{array}{ll}
N_{s} \sim N_{h v} d_{s} /\left(\sigma_{r}{ }^{2}+\sigma_{y^{\prime}}{ }^{2}\right)^{\frac{1}{2}} & \text { or } \\
N_{s} \sim N_{h v} d_{s} / M_{s}\left(\sigma_{y}{ }^{2}+\ell_{s} \sigma_{r}-\right)^{\frac{1}{2}} \simeq N_{h v} d s / M_{s} \sigma_{y} & \text { where }
\end{array}
$$

$M_{g}$ is the optical (de)magnification factor, $d_{g}$ is the slit width and $\&_{s}$ is the azimuthal source extent. Siace for a multiplicity of beam lines it is desirable to be able to optinize the transmitted photon flux in either case, it is desirable, from a synchrotron radiation source optimization point of view, to minimize both $\sigma_{y}$, and $\sigma_{y}$ simultaneously, hence to minimlze tiise beam vertical emittance, $\varepsilon_{y}$. For a practical electron storage ring magnet structure the vertical beam emittance is determined by the magnitude of radial-vertical coupling of the betatron motion, whlch results in a typlcal magnitude of $\varepsilon_{y} \simeq 10^{-2} \varepsilon_{x}$ (i.e. it is difficult to reduce the coupling coefficient below $\simeq 0.1)$. As a consequence, in practice, minimm vertical beam emittance is obtained with the reduction of the horfzontal heam emfttance. Therefore, also in this case aximum source brightness (simultaneous 
reduction of $\varepsilon_{x}, \varepsilon_{y}$ ) is the relevant criterfon for optimum synchrotron radiation source design.

\section{Electron Storage Ring Structure}

The beam emittance $\varepsilon_{x}$ (and "coupled" with this $\varepsilon_{y}$ ) In an electron storage ring is the result of the longltudinal and transverse oscillation amplitude damplng assoctated with the mean energy loss due to the emission of synchrotron radiation, limited by the random excitation of the oscillation amplitudes due to the discrete quantum nature of the photon emission. Its equilibrium value is dependent on electron beam energy and the parameters of magnet ring structure. The analysis of the relationshtp of the beam emittance and storage ring lattice structure parameters mis be found In Sands ${ }^{2}$. From thls, sssuming a well aligned magnet structure and no momentum dependent effects:

$$
\varepsilon_{x}=c_{q} \gamma^{2} I_{5} /\left(I_{2}-I_{4}\right), \quad \varepsilon_{y}=k^{2} \varepsilon_{x} \quad \text { with }
$$

$C_{q}$, a constant and the synchrotron radiation Integrals, $I_{1}$, defined as

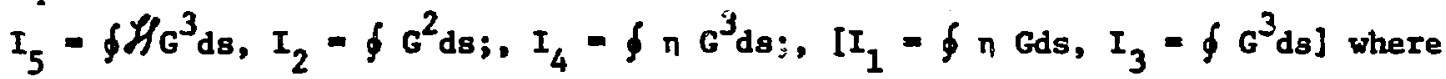
$\mathcal{H}$ is the dispersion function invariant $\mathscr{H}^{\star}=\bar{\gamma} \eta^{2}+2 \bar{a} n \eta^{\prime}+\bar{\beta} n^{\prime 2}$ with $n(s)$, the electron orbit dispersion function and $G(s)$, the curvature function.

*Analogous to the Courant-Snyder invariant, however, $\mathscr{H}$ is invarlant only through drift spaces and focusing elements of the lattce, it changes through dipoles. 
For a sepazated function, 1so-magnis, ic, storage ring the curvature function $G(s)=1 / \rho$, resulting in $I_{5}=(\oint h \mathrm{ds}) / \rho^{3} ; I_{2}=2 \pi / \rho ; I_{4}=2 \pi \alpha_{p} R / \rho^{2}$ with $\rho$ the magnetic bending radius and $\alpha_{p}=(\Delta C / C)(\Delta p / p)$, the storage ring momentum compaction function. Since $\alpha_{p} \ll 1$ it follows that

$$
\left.\varepsilon_{x}-c_{q} r^{2}\left[(\oint \not{H} \mathrm{ds}) / 2 \pi p^{2}\right)\right]
$$

The bracketed part 18 dependent on the magnet structure parameters only, therefore, for various synchrotron radiation structures, designed for different electron team energies $(\gamma)$, a "IIgure of merit"characterizing the storage ring lattice structure may be defined, given by

$$
(\$ / / \mathrm{ds}) / \mathrm{o}^{2}
$$

This quantity has been evaluated for a number of storage ring structures efther in existence or under construction at the present time for dedicated synchrotron radiation sources. This is sumarized in Fig. I where the lattice structure functions are given for these sources together with the magnitude of the lattice figure of merit. For lower energy storage rings the latter quantity tends to be larger because the objective of $\operatorname{small} \varepsilon_{x}$ (and $\varepsilon_{y}$ ) must be balanced, for econoritcal reasons, by the objective of highest photon energy $\left(\varepsilon_{\text {crit }}=c_{1} \gamma^{3} / p\right)$ for a particular structure, whout going to higher electron energy $\left(P_{r f} \in C_{2} \gamma^{4} / \rho\right)$, 1.e. a shift to shorter wavelengths is obtained with the use of smaller $\rho$ values In the magnetic structure. While taking this aspect Into account, it follows, however, that a superior synchrotron radiation source lattice structure is obtained for a ring for which Hds has a ninimum value. This is optimally achieved by use of basic achromatic bend sections separated by zero momentum dispersion sectlons. An added requirement at the origin location of the scurce is the need for miajum B values for optimum local source brightness. This combination of 
FIGURE 1. STRUCTURE COMPARISON OF SOME REPRESENTATIVE SR SOURCES

SPEAR, 3 GEV, $\mathrm{e}^{+} \mathrm{e}^{-}$, "FOO"

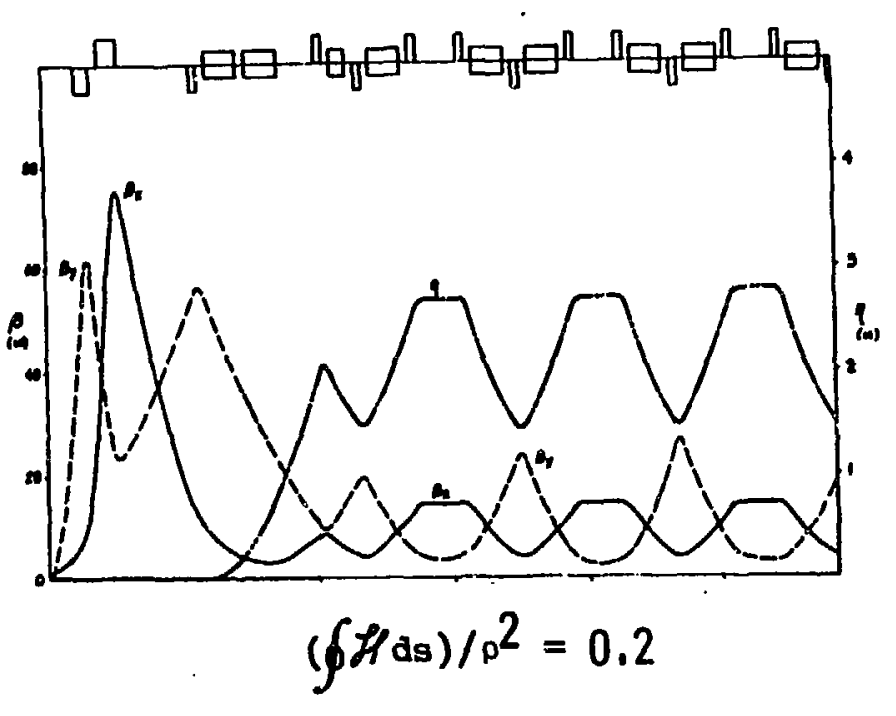

ALADDIN, 1 GEV, SR, "DOUBLET"

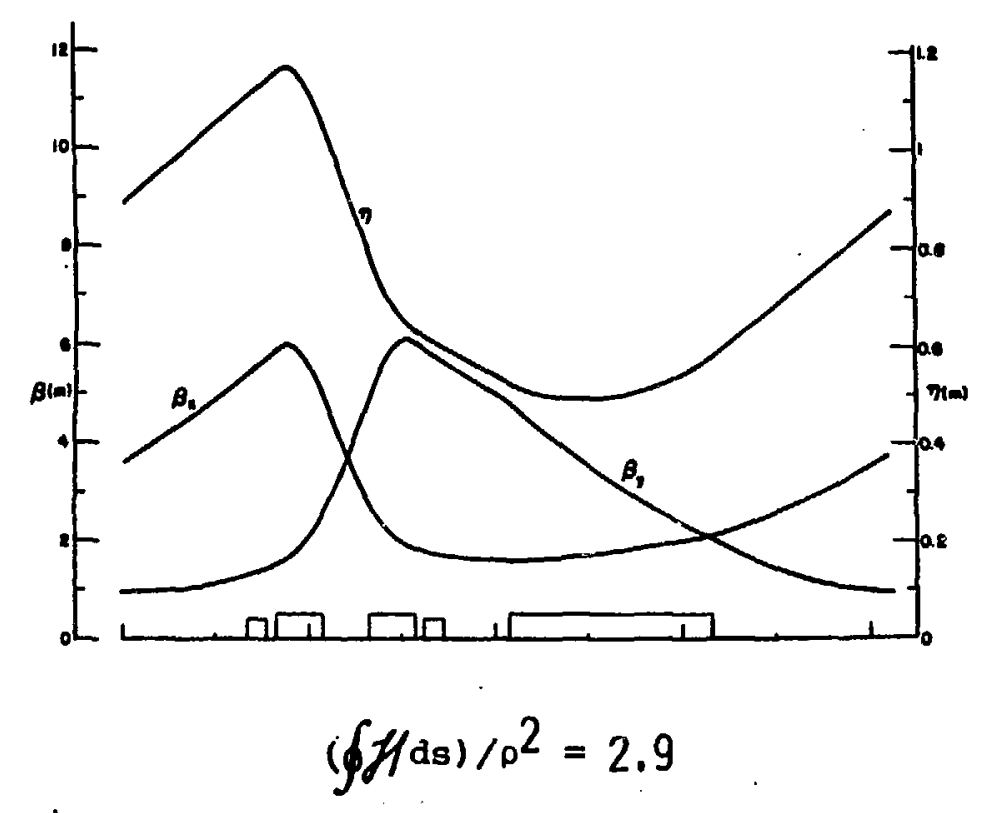

PHOTON FACTORY, 2.5 GEV, SR, "FODO"

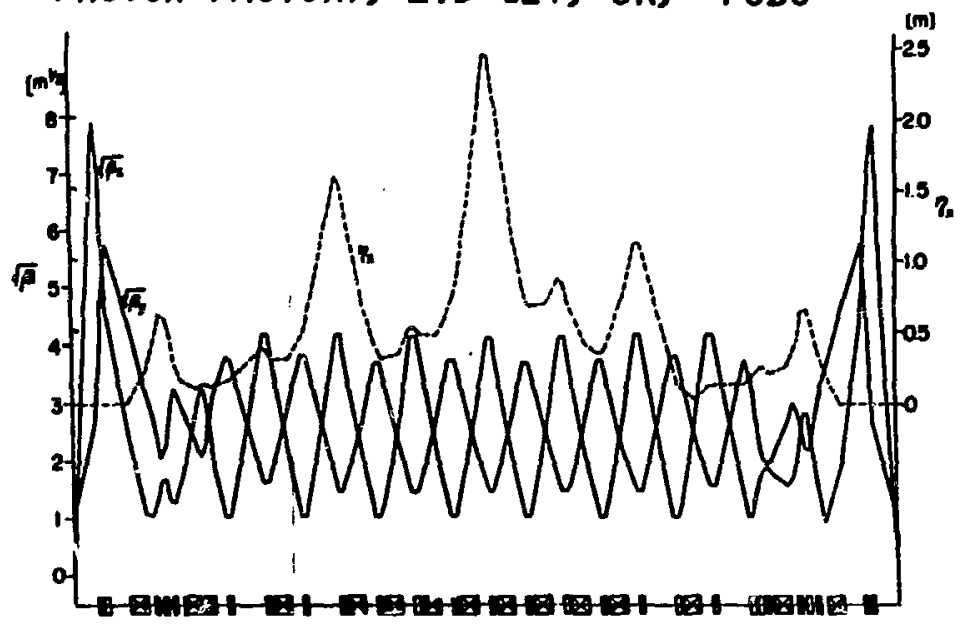

$$
(\oint y d s) / p^{2}=0.28
$$

NILS, 2.5 GEV, SR, "TRIPLET-ACHRO.B."

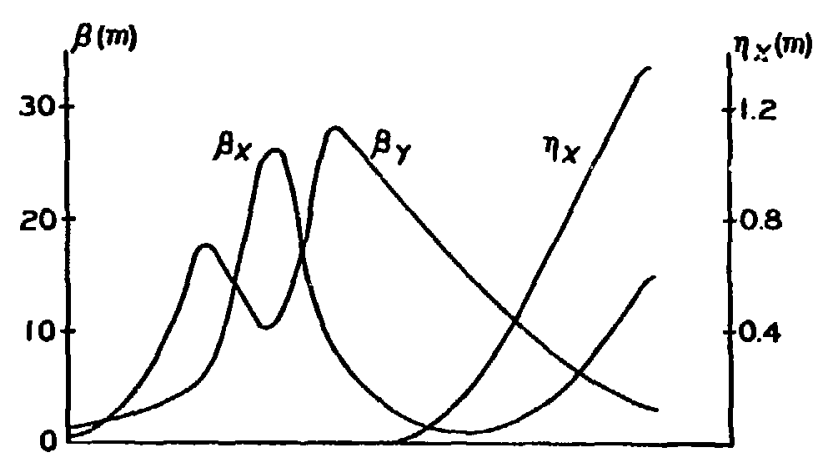

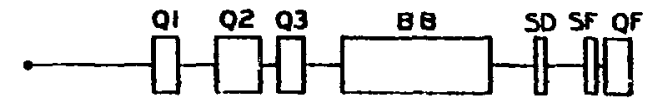

$(\oint \mathcal{H}$ as $) / \mathrm{p}^{2}=0.055$ 
criteria has been met successfully, with a minimum number of magnet components in the design of the NSIS storage rings, 3,4 of which the 2 -ray lattice, together with basic parameters, Is given in Fig. 2.

Choice of Electron Accelerating Sygtem Parameters

For dedicated synchrotron radiation sources, a number of storage ring optics operational modes are of interest. In case of high resolution spectroscopy a high brightness source (four dimenstonal phase space density, $\mathrm{N}_{h v} / \sigma_{y}{ }^{2} \sigma_{x} \sigma_{y}$ ) 18 desired. This, as shown above, requires a small $\varepsilon_{x}$ or \$fds value. This tends to result also in a small momentum compaction, $\alpha_{p}$, value, with as a consequence a smaller bunchlength $\left(f_{b} \propto a_{p}^{\frac{1}{2}} f^{-1}\right.$, see below). As a result a high volume density Is obtalned $\left(I / \sigma_{x} \sigma_{y} \sigma_{T}\right)$, which limlts the source brightness lifetime because of the so-called Touschek effect, a loss of particles from longitudinal phase stability due to intrabunch electron scattering. In this case a lower if frequency value for the electron accelerating system would be favorable gince this promotes larger bumch length values. Alternatively, for dynamic measurements, using synchrotron radiation sources, sample fllumination duration 18 of 1mportance. Typicaliy, for fluorescent lifetime messurements bunch length values as low as 0.1 nsec (or $\ell_{b} \simeq 3 \mathrm{~cm}$ ) may be desired in specific cases, possibly at a cost in total photon flux available in order to maintaln acceptable sourse brightness IIfetime. Consequently, factors affecting bunch length are of Interest here, such as If parameters, use of harmonic accelerating cavity, lattlce structure parameters, etc. Extending now the work by Saxon and Schwain ${ }^{5}$ on the choice of the rf frequency for the Daresbury storage ring, a few relationshlps are given here separatIng out specifically the bunch length dependency on structure parameters and on If: parameters.

The electron beam bunch length, for equilibrium energy spread is given $b y^{2}: \quad \quad \ell_{b}=2 c \sigma_{T}=\left(c a_{p} / \pi \bar{r}_{s}\right)\left(\sigma_{E} / E\right)$ 


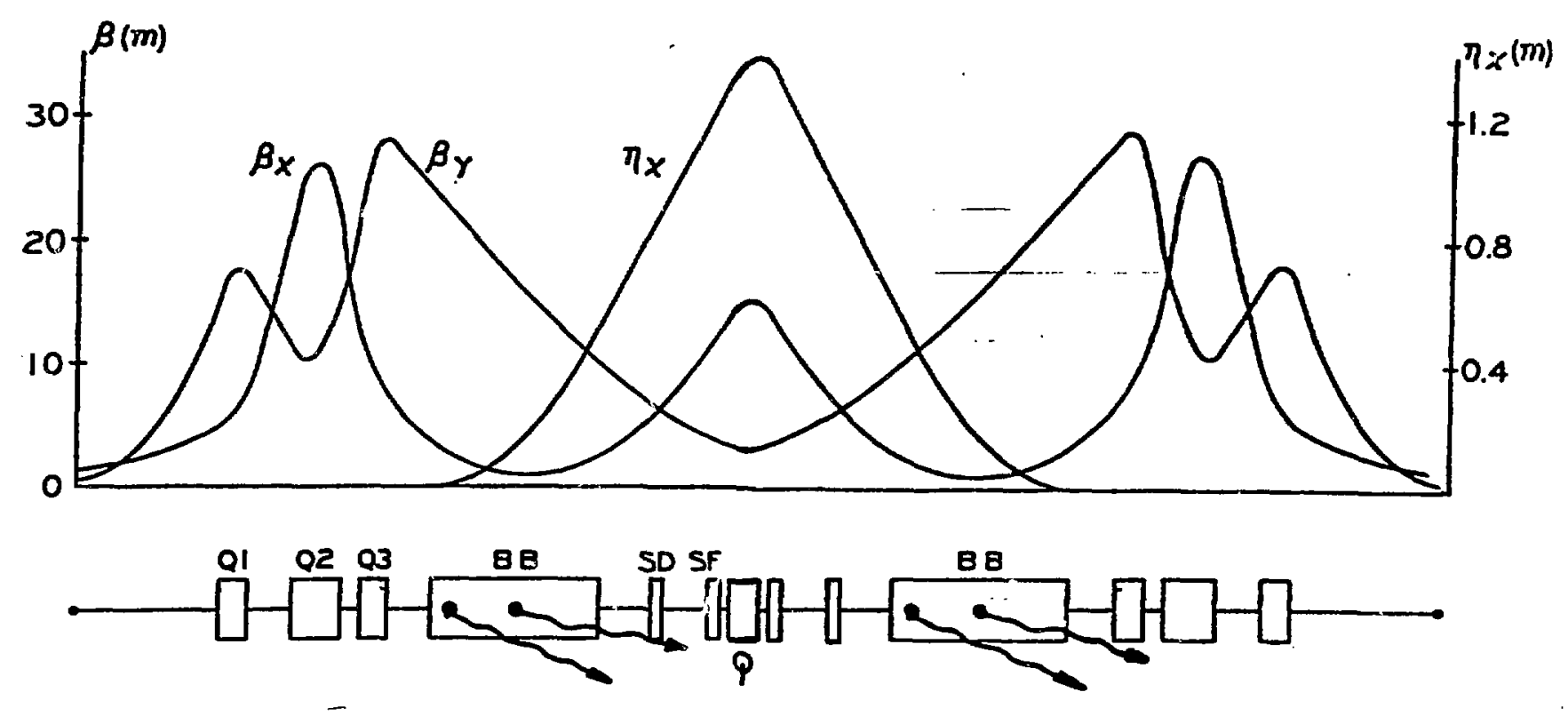

FIGURE 2

2.5 GEV LATTICE STRUCTURE OPTIMIZED AS A SYNCHROTRON RADIATION SOURCE

$$
\begin{aligned}
& -\rho_{M}=6.9 \mathrm{M} ; \hat{B}=1.2 \mathrm{~T} ; L_{B}=2.7 \mathrm{M} ; C=170 \mathrm{M} \\
& v_{X}, \nu_{y}=9.7,5.7 ; \alpha_{P}=0.0065
\end{aligned} \quad\left\{\begin{array}{l}
\varepsilon_{x}=810^{-8} \mathrm{M} \cdot \mathrm{RAD} . \\
\varepsilon_{y}=810^{-10} \mathrm{M} \cdot \mathrm{RAD} . \\
\left(\sigma_{\varepsilon} / E\right)=8.210^{-4}
\end{array}\right.
$$


where $\sigma_{\tau}$ is the bunch duration, $f_{s}$ is the synchrotron oscillation frequency and $\left(\sigma_{\varepsilon} / E\right)$ is the standard deviation energy spread of the bunch. The quantity $f_{6}$ is geven by

$$
f_{s}=f\left(\alpha_{p} \nabla \cos \varphi_{s}\right) /(2 \pi h E)
$$

where $f$ is the frequency of the electron accelerating field, $h$ is the harmonic number (number of possible bunches in the Iing), $V$ is the rf voltage anplitude and $\varphi_{\mathrm{g}}$ is the synchronous phase angle. It $1 \mathrm{~s}$ of Intexest now to separate bunch length dependency on lattice structure parameters and on the accelerating system parameters. This is simply done by making use of the expressions for the synchrotron radiation integrals. It follows:

$$
\begin{aligned}
& \ell_{b}=\left[\frac{4 \mathrm{ec}}{\left(m c^{2}\right)^{2}} \frac{c_{q}}{c_{\gamma}}\right]^{\frac{1}{2}} \cdot\left[\frac{I_{1} I_{3}}{I_{2}\left(2 I_{2}+I_{4}\right)}\right]^{\frac{1}{2}} \cdot\left[\frac{1}{f^{\frac{1}{2}}\left(q^{2}-1\right)^{\frac{3}{4}}}\right] \cdot \frac{1}{E^{\frac{1}{2}}} \text { where } \\
& c_{q}=3.810^{-13} \mathrm{~m} ; C_{\gamma}=8.8510^{-5} \mathrm{~m} \mathrm{GeV} ; q=\left(e \nabla / v_{0}\right)=\left(\sin \varphi_{s}\right)^{-1}
\end{aligned}
$$

with $U_{0}$ the synchrotron radiation losi per orbit and $q$ the if overvoltage factor; or

$$
\begin{aligned}
& l_{b}=\text { Const. Cstructure } \cdot F_{\text {If }} \cdot E^{-\frac{1}{2}} \text { where } \\
& F_{r f}=f^{-\frac{1}{2}}\left(q^{2}-1\right)^{-\frac{1}{4}} .
\end{aligned}
$$


A relationship $q=F(f)$ may now be found by considering the following: The quantum nature of the energy loss due to synchrotron radiation requires a large phase stable "bucket" area in order to prevent electron loss from the longitudinal phase stable area. A "quantum life time" may be associated with this loss process. Demanding now a (minimum) Iife time value of $\tau_{q}=100 \mathrm{hrs}$, the magnitude of $q$ mag be quantized ${ }^{2}$, and leads to a relationship $q=F(f)$, which for values of $q>1$ can be approxfmately written (for specific Isomagnetic-separated function lattice structures) as:

$$
q \cong c_{1}\left(a_{p} R / E\right) f+c_{2}
$$

indicating the need for larger of overvoltage factors when using higher acceleratIng frequency values (typlcal: NSLS; $f=50 \mathrm{MHz}, \grave{q} \cong-1.3 ;$ SRL; $f=500 \mathrm{zHz}, \underline{q} \cong 7.5$ ). As a consequence now, an approxinate expression for $t_{b}$ may be written as:

$$
\ell_{b} \cong \text { Const. } \cdot C^{\prime}{ }_{\text {structure }} \cdot \mathrm{f}^{-1} \mathrm{E}^{-\frac{1}{2}}
$$

stating the inverse proportlonality of bunch length and frequency of the accelerating sybtem. This is further fllustrated in Fig. 3 where it is Indicated that a choice of a low accelerating frequency value would be unacceptable from the point of view of dynamic spectroscopy uniess this would be matched with an optimized design of the storage ring lattice structure (typical calculated values (ignoring bunch "blowup": NSLS; $f=50 \mathrm{MHz} ; 2 \sigma_{T}(q=1.3) \cong 0.35 \mathrm{nsec} ; \mathrm{PF}, f=500 \mathrm{MHz}, 2 \sigma_{T}(q \simeq 4.5)$ $\simeq 0.13 \mathrm{nsec}$ ). With a lower frequency choice, a higher overvoltage factor than that required for acceprable quantum lifetime is readily achievable, reductng thereby further the bunch length value. In addition, by suftable use of a harmonic if cavity a further reduction of bunch length can be achleved.

The cholce of accelerating frequency has further specific consequences in terms of the minimum shunt impedance of the accelerating structure (higher $q$ value or higher frequency unfavorable); the beam Induced voltage in the accelerating 


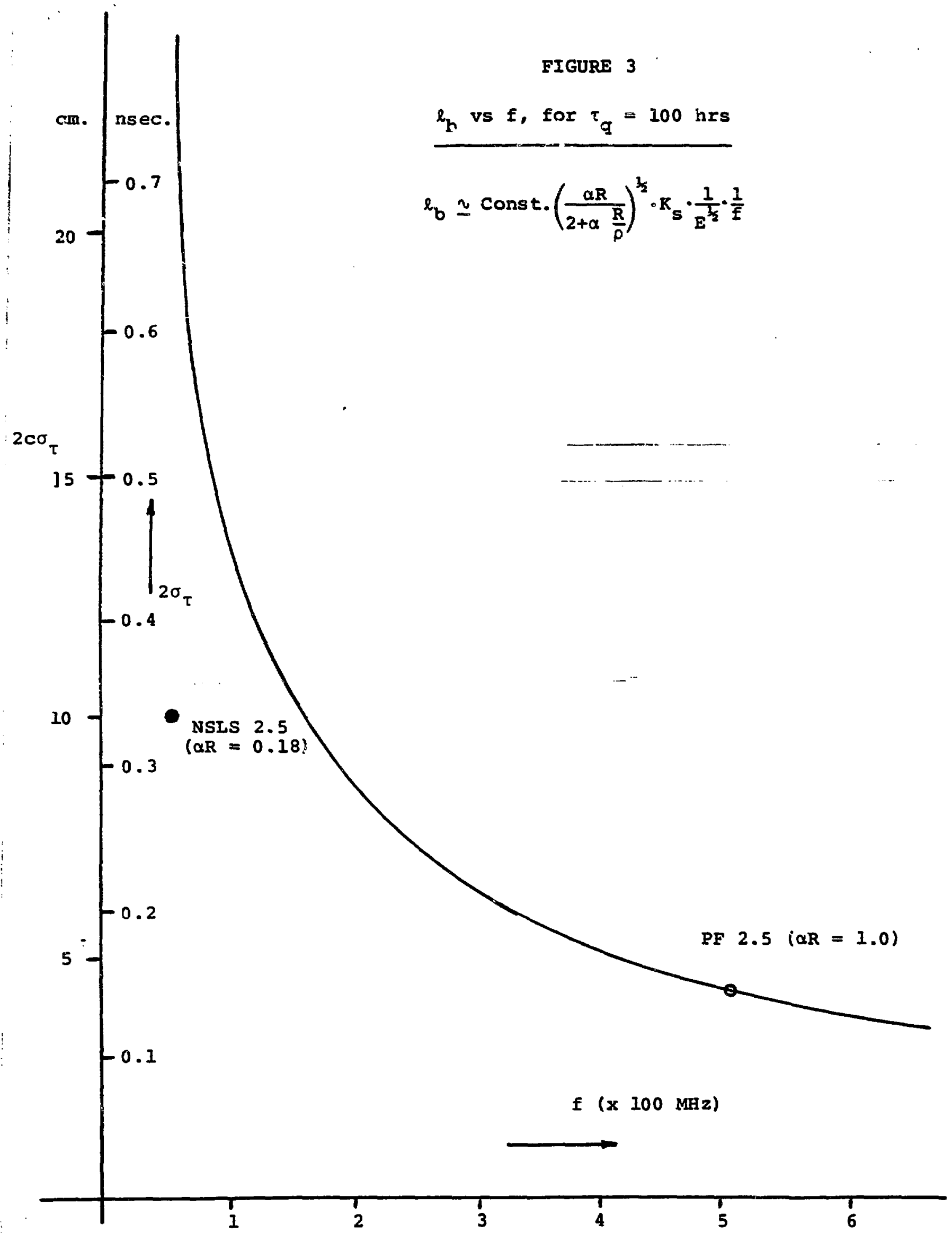


structure (again lower q value desired); $v_{g}$, the synchrot:ron osclllation wave number and in terms of $10 n$ trapping in the potential well of the circulating electron beam. Here, only the latter two aspects will be further mentloned. The synchrotron oscillation wave number, for a "non-distorted bucket", Is given by

$$
v_{s}=\left(f_{g} / f\right)=\left[\left(f_{s} / f_{0}\right)\left(\alpha_{p} \nabla \cos \phi_{B} / 2 \pi E\right)\right]^{\frac{1}{2}}, \text { whIch }
$$

can be written as

$$
v_{s}=\left[C_{\gamma} / 4 \pi^{2} e c\right]^{\frac{1}{2}} \cdot\left[I_{1} I_{2}\right]^{\frac{3}{2}} \cdot F_{r f}-1 \cdot E^{3 / 2}
$$

Assuming again a fixed quantum lifetime, $\tau_{q}$, value, and using the above given $q$ - $F(f)$ relationship, it follows that:

$$
v_{s} \cong \text { Const. } \rho^{-x_{x}}\left(\alpha_{p} R\right) E \mathbf{f}
$$

In general, it is desirable to avold high $v_{8}$ values, because of potential betatronsynchrotron resonances, 1.e. sidebands of the principal lattice resonances given by $v_{B}-n v_{s}-m$ (low $n$ values, unfatorable). With accelerating frequency values of $f \simeq 500 \mathrm{MHz}, v_{s}$ values of $\sim 0.1-0.01$ will be encountered, resulting in greater constraints on the cholce of permissible $v_{x}, v_{y}$, the betatron oscillation frequency, values. WIth a cholce of $f=50 \mathrm{MHz}, v_{s}$ values of less than 0.01 are readily present, avolding thereby the problem of lossy resonant sidebands.

As a result of residual gas ionization with resultant ion acceleration towards the electron beam, Ion trapping in a transverse oscillatory motion can occur. This causes partial neutralization of the electron beam space charge and results in a tune shift $\left(\Delta v_{x, y}\right)$ of the transverse oscillatory motion. In general, ime shifts greater than $0.25 \mathrm{~V}$ units are considered harmful because of crossing Into and through structure resonant stopbands. The standard remedy for this problem Is the use of electrostatic clearing fields which accelerate the fons into the 
vacum chamber wall. A very serlous drawback of this procedure in high intenalty storage rings is the Increase of the vacuum envelope wall 1wpedance with the introduction of the clearing electrodes, aggravating thereby the potential for instabllity thresholds at lower beam current values.

The mechanism of Ion trapping has been treated quantitatively by Rohaupt. 6 Depending on the magnttude of the electron beam intensity, the 1ons are accelerated towards the potential well of the electron beam and elther trapped into a transverse oscillatory motion around (through) the electron beam or galn sufficlent trangverse energy to traverse through the potentlal well to be logt on the vacuum chamber envelope. Stable trangverse oscillatory motion of the lons occurs if $I_{e^{-}}<I_{\text {st }}$ with

$$
I_{s t}=310^{7} \mathrm{~A} \sigma_{x} \sigma_{y} \ell_{b b}^{-2}\left(1+\sigma_{y} / \sigma_{x}\right)
$$

where

A Is the Ion mass number and $h_{b b}$ the bunch separation distance, f.e. for $I_{a^{-}}>I_{s t}$ the fons are accelerated into the wall and no partial beam neutralization occurs. Since $b_{b b}=c / f$ (for a oystem where all avallable rf "buckets" are filled), lower $I_{s t}$ values are encountered with the choice of a loser accelerating frequency. If $I_{s t}$ is sufficientig low, clearing electrodes will not be needed, since the stable (for fon trapfing) current value w111 read1ly be traversed during the injection process. (If need be, empty "buckets" could be used, to Increase \&b temporartly during the infection process.) To Lllustrate this, some typical values are given here for the NSLS storage ring, as follows: $f=50 \mathrm{kHz}, b_{\mathrm{bb}}=5 \mathrm{~m}, \mathrm{I}_{\mathrm{st}} \simeq 0.1 \mathrm{~A}$ for $\mathrm{H}_{2}, \mathrm{I}_{\mathrm{st}} \simeq 1.5 \mathrm{~A}$ for $\mathrm{Co}$. With design parameters for this ring of $0.5 \mathrm{~A}, 2.5 \mathrm{GeV}$ or $1.0 \mathrm{~A}, 2.0 \mathrm{GeV}$ the stable current value $\left(I_{\mathrm{gt}}\right)$ is readily exceeded, taking into account the presence of lighter Ion species only. No clearing electrodes are incorporated in this ring, suggesting, however, the need for spectal precautions to prevent contamination from heavier ion specles (hydrocarbons). If, Ingtead a high 
frequency accelerating system would be adopted here, the following values would be encountered: $f=500 \mathrm{MHz}, l_{b b}-0.5 \mathrm{~m}, I_{s t} \simeq 3.0 \mathrm{~A}$ for $\mathrm{B}_{2} ; I_{s t} \simeq 150 \mathrm{~A}$ for $\mathrm{Co}$, Indicating the Imposibility of exceeding the I'n trapping stable current. Consequently, In this case clearlag electrodes wrould be required in the ring structure.

Other aspects entering into the cholce of accelerating system parameters are the certain presence of spectfic longltudinal and transverse beam instabllitles. This will not be addressed.here, other than to state that in general with the use of low frequency accelerating systems, current linitations are escountered assoclated with single bunch Instabllities due to the seif interaction of the electron bunch with Its short range wake flelds induced in the beam envelope. Alternatively, In case of high frequency accelerating wyatems, with 1 ts mall bunch separation, current limitations are more likely to be encountered because of difficult to control multiple bunch Instabilities assoclated with bunch to bunch long range wake fleld interactiong.

\section{Special Lattice Structure Sections}

In order to extend the wavelength reglon avaliable from a synchrotron radiation source or Increase its photon flux avallable in a specific narrow wavelength region, special components have been developed such as the superconducting "wigsler" and the "undulator". Both devices are orbit perturbators with no net electron orbit deflection. Recaling that $\varepsilon_{c} \propto \gamma^{3 / \rho}$ and $P_{\text {rf }} \times \gamma^{4} / \rho$, a high field wggler provides for an economical way of extending the avallable photon spectrum to higher energy values. Assuming the use of $6 \mathrm{~T}$ peak field values, a factor of approxdmately 5 (or more) in critical energy may be obtalned. Therefore, state of the art dedicated synchrotron radiation sources will have an adequate number of long straight sections in the magnet lattice structure for hard wavelength wiggler sources. The objective of optimim source brightness leads to the criteria that $B_{x}, B_{y}$ are small in magnttude in the center of the wiggler straight section 
and also that the local dispersion function, $n$, Is preferably equal to zero since B $\alpha\left(\sigma_{x} \sigma_{y}\right)^{-1}$ where $\sigma_{y}=\left(\beta_{y} \varepsilon_{y}\right)^{\frac{1}{2}}$ and $\sigma_{x}^{2}=\left(\beta_{x} \varepsilon_{x}+n^{2} \sigma_{\varepsilon}^{2} / E^{2}\right)$. A more significant aspect of the oujective to design for zero dispersion in the wiggler straight section is the degire to avold antidampling and enlargement of the bean emittance. The magnicude of this has been calculated for a typlcal structure (NSLS, $x-r a y)$. By straightforward circumferential. Integration of the digpersion Invariant in the lattice with and without wigglers it is found that ${ }^{7}$

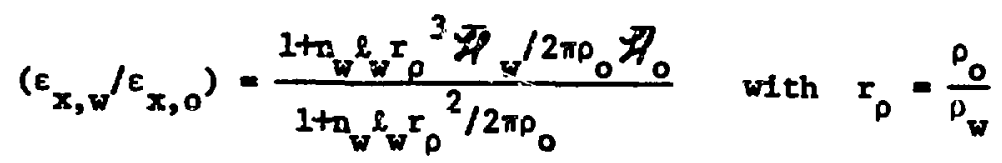

$\bar{H}_{0}$ is the average value of $\mathscr{H}_{1 a}$ the structure without wggler magnets and $\dot{\mathscr{H}} w$ is the average value of $\mathscr{H}$ over the wiggler domain only. Further, the mean value of the dispersion invariant in a single pertod of the wiggler structure is given by ${ }^{8}$ $\bar{y} \simeq H_{1 n}+B_{x} \theta / 3$ where $\theta$ is the orbit arc extend per one quarter yiggler period. Inserting five wigkilers with the following parameters, $\ell_{w}=3 \lambda_{w}, \lambda_{w}=0.14 \mathrm{~m}$, $\hat{B}=5 \mathrm{~T}, \mu_{w}=1.6 \mathrm{~m}, \theta=16.7 \mathrm{mrad}, \rho_{0}=6.8 \mathrm{~m}$, in a straight section of $\bar{\beta} \simeq 1 \mathrm{~m}$ and non-zero disperzion with $\overline{\mathscr{H}}_{\mathrm{v}} \simeq \overline{\mathscr{H}}_{0} \simeq 0.06 \mathrm{~m}$, an emtttance growth $\left(\varepsilon_{w, x} / \varepsilon_{x, 0}\right) \simeq 2.4$ results. Alternatively, If these wigglers would be Inserted In zero dispersion straight sections, then $\eta_{w}=910^{-5} \mathrm{~m}, Z_{0}=0.06 \mathrm{~m}$ and the equlibrium emittance would be reduced as $\left(\varepsilon_{x, w} / \varepsilon_{x, 0}\right) \simeq 0.6$.

An undulator ${ }^{9}$ is a structure consisting of many low field wigglers in sequence either in the form of a flat pole wiggler or helical wiggler. Its basic objective is the generation of high photon flux, quasi monochromatic peaks in the synchrotron radiation frequency distribution, given by (median plane):

$$
\lambda_{k}=\lambda_{w}\left(2 k \gamma^{2}\right)^{-1}\left(1+k^{2}\right) \quad \text { with } k=0.1 B \lambda_{w}(k G, c m) \text { and } k=1,3,5 \ldots
$$


The total photon flux avallable from the undulator 18 proportional with $n_{w}$, the number of undulator periods, however, the central brightness is proportional with $n^{2}$, or $^{10}$

$$
\left.\frac{d P}{d \sqrt{2}}\right|_{\theta=1}=\frac{2 n_{w}{ }^{2} e^{2} \gamma^{2} x^{2}}{c\left(1+k^{2}\right)^{2}}
$$

As shown by Rincald ${ }^{11}$ this central brightness (and the spectral line width $\left.(A \lambda / \lambda) \simeq 1 / \mathrm{n}_{\mathrm{w}} \mathrm{k}\right)$ can only be obtalned if the structure functions in the undulator straight section satisfy the criterion that $\sigma_{x^{\prime}} \simeq \sigma_{y^{\prime}} \simeq 1 / \gamma \sqrt{n_{w}}$. This requires, for a typical-synchrotron radiation-lattice structure (e.g. NSLS-700 KeV ring), that the local $\beta$ values: In the undulator straight-gection are -modest In magnitude $\ldots$. ( $10 \mathrm{~m})$ rather than low $(\beta \simeq 1 \mathrm{~m})$. for optimum undulator :brightness value. Also in this case is it desirable to locate the undulator in a straight section with zero local dispersion value in order to obtaln mestim sours a brightness.

The foregoing synchrotron radiation source optimization aspects have-been taken Into-account-in the design of the two electron storage rings-comprising the National Synchrotron Light Source, of which the principal parameters are:

$\begin{array}{lll} & \text { X-ray Source } & \text { VUV Source } \\ \text { Current, energy (A, GeV) } & 0.5 ; 2.5 & 1.0 ; 0.7 \\ \text { CIrcumference (m) } & 170 & 51 \\ \lambda_{c}(\AA) & 2.5(0.5)_{w} & 31.6 \\ \text { Enfttance, } \varepsilon_{x}(\mathrm{~m} \text {-rad) } & 810^{-8} & 910^{-8} \\ \text { SR Power }(k F) & 300(5 \text { wgglers) } & 12 \\ \text { Source } 4 \sigma_{x}, 4 \sigma_{y} & 0.5 \times 1.5 \text { (arc) } & 0.4 \times 1.2 \\ \left(\mathrm{~m}^{2}\right) & 0.1 \times 0.9 \text { (w1ggler) } & \text { (arc) }\end{array}$

A more complete presentation of the NSLS bast; design and parameters is given elsewhere. 12 


\section{REFERENCES}

1. G. K. Green, "Spectra and Opt16s of Synchrotron Radiation", BNL Report 50522, (1976).

2. M. Sands, "The Physics of Electron Storage R1ngs", SLAC Report 121 (1970).

3. L. Blumberg, J. Bittner, J. Galayda, R. Heese, S. Rrinsky, J. Schushman, and A. van Steenbergen, 'National Synchrotron Light Source VUV Storage R1ng", IEEE Trans. Nucl. Sc1. NS-26 (1979) 3842-4.

4. S. Krinsky, L. Blumberg, J. Bittner, J. Galayda, R. Heese, J. Schuchman, and A. van Steenbergen, "Design Status of the $2.5 \mathrm{GeV}$ National Synchrotrox Lfght Source X-ray RIng", IREE Trans. Nual. Sct. NS-26 (1979) 3806-8.

5. G. Saxon and T; E. Swa1n, "The Choice of Radiafrequency far the Daresbury -Storage RAng", Report DLSRF-R6, Daresbury, A.K. (1975).

6. R. D. Kohaupt, "Ion Cleaning Mechanism in the Electron-Positron Storage Ring Dor18", Report DESY H1-71/2 (1971).

Z. J. M. Patergon, J. R. Rees; H. Wiedemann, "Control of Bem-Size and-Rolarizatton TIme in PEP", Report Spear-186, PEP-125, July 1975.

8. A. Button, Pairt. Accel. 7, No. 3 (1976) 163.

9. H. Motz, et al., J., App1. Phy8. 26 (1953) 826.

10. D. F. Alferov, et a.l., Sov. Phys. Tech. Phys. 18. (1974) 1336.

11. B. Rincaldy J. App1. Phys. 48 (1977) 2684.

12. A. van Steenbergen, Proc. Nat'1. Conf. on Synchrotron Radiation Instrumentation, Jume 1979, Nucl. Instrum. Methods (1980) 